\title{
Stamping Tools for Sheet Metal Forming: Current State and Future Research Directions
}

\author{
Carl-Johan JONSSON ${ }^{1}$, Roland STOLT and Fredrik ELGH \\ Industrial Product development, Production and Design, School of Engineering, \\ Jönköping University, Sweden
}

\begin{abstract}
Sheet metal forming tools play an important role in the manufacturing of many products. With shorter product life cycles and demand for shorter time to market for new products, the process for design and manufacturing of stamping tools becomes a critical part. Stamping dies are often designed and manufactured by smaller, specialized companies. For a tooling company, knowledge and experience is an important competitive advantage. Traditionally the design process has been characterized by being based on few key individuals with much experience and craftsmanship. To stay competitive in this market there is a need for more efficient processes, systems, tools and supports in order to become more industrialized. This paper presents results from a study of the state of practice in industry within progressive stamping tool design as well as a review of relevant literature. The design and manufacturing processes for stamping dies in six companies have been investigated through semi-structured interviews, from which the main challenges in the current state for the companies are identified. The results from the interviews was analyzed and compared to the established concepts and frameworks of methods found in the literature review. The results and analysis points in the direction of efforts needed in supporting the formalization and reuse of information and knowledge from previous tool projects and production, especially during the critical steps of tool process planning and creating the tool layout.
\end{abstract}

Keywords. Sheet metal forming, Stamping, Dye design, State of practice, State-ofthe-art

\section{Introduction}

Sheet metal forming (SMF) tools play an important role in the manufacturing of many products. The design of the tool affects the component properties, production rate, material utilization, post processing, quality and rejection rates. Tool design and manufacturing are often carried out by smaller and more specialized companies who delivers a complete tool ready for production of a sheet metal component. The component is often designed by larger companies, original equipment manufacturers (OEM), while the actual manufacturing of the component can be done by a subsupplier. Customization is becoming more important for companies for delivering higher customer value and getting more efficient resource utilization in products. With higher product variety, that results in lower production volumes per tool. But stamping

\footnotetext{
${ }^{1}$ Corresponding Author, Mail: carl-johan.jonsson@ju.se.
} 
tools for SMF are quite expensive and require large volumes to be an economically good choice. OEMs demand shorter lead times to be able to have a shorter time to market. At the same time, there is a need to be able to quickly adapt to new requirements and customizations that can appear late in the product development process. Another aspect to this is that the adoption of the concurrent engineering paradigm has the consequence that tool design starts with uncertain or incomplete information and changes are to be expected [1]. In a competitive and global market, tooling companies submits quotations on numerous requests annually. To stay competitive in this market and enable higher product variety, there is a need for more efficient processes, systems, tools and supports to become more industrialized and enable companies to improve their ability to introduce new technology.

This paper presents results from the first part of a research project that is aimed at addressing challenges in designing SMF tools. The project uses the design research methodology (DRM) [2] and the study described here is part of the research clarification step. It is important to first understand the environment that improvements are to be implemented in. The first step in the project is therefore, to map the state of practice and identify the main challenges from the perspective of the tool designers. Some shorter, general descriptions of the tool design process can be found in various handbooks [3] but give little insight to the current state of practice. Also, traditionally the process of designing and manufacturing SMF tools has been characterized by being based on few key individuals with much experience, and craftsmanship. In this study, tool designers at six companies have been interviewed to get their qualitative view on what their process of tool design looks like, what design supports are used, how time and cost are distributed in the process and what their main challenges are. To be able to identify challenges and address them, the focus in this work is more on closer collaboration with the companies to get a more in-depth view, rather than broad.

In the following sections a review of relevant literature is made to overview the state-of -the-art for methods and supports in tool design. This is followed by the results from the interviews and an analysis comparing findings from the interviews with existing research from the literature review. Lastly the conclusions and a summation of future research directions and areas is made.

\section{Review of the literature}

The review of the literature was broken down in four sections: Design automation Simulation and material science and Platform approaches. Additionally a summary of the review performed by Kolhatkar and Pandey [4] on use of sensor technology and online monitoring in sheet metal tooling is found in the last section.

\subsection{Design Automation}

There have been many different attempts to automate the design of progressive dies for sheet metal stamping. Kumar and Singh [5] developed a knowledge-based system (KBS) for assisting the tool designer and automating parts of the process. Their system is implemented in AutoCAD and structured in different modules. The modules serve different purposes and solve problems using different methods. During the use of these modules different tasks are carried out. These are: flattening of the sheet metal part, nesting (to minimize scrap and balance the punch forces), creating the punch shapes, 
creating the bending die shapes, sequencing the punching and bending operations, deciding how and where pilots should be used, making the process plan including idle stations, detail design of all components of the tool, choose proper material for parts.

Different methods, systems and algorithms have been developed by researchers to automate or optimize the results of some of these tasks. Most commercial CADsystems are able to flatten shapes based on developable surfaces. And some can flatten more complex shapes. Automatic nesting and piloting have been done in [6] and later in [7]. Their nesting algorithms are similar in that they are both placing two copies of the flattened shape next to each other and rotate the shapes in small increments and identifies eventual collisions and repositions the parts. Then for each rotation increment the scrap rate is calculated and lastly the angle with the minimum scrap is chosen. Notable here is that there are other criteria besides scrap that are of interest for optimizing towards, for example force balance of the punch and bending operations. But these are not considered. The pilot selection has different approaches as the algorithm [6] gives the designer areas where direct, indirect or semi-direct pilots could be placed and the approach in [7] can give specific points for were to place the pilots.

Sequencing of operations in the progressive die have been approached from different angels. Li et al. [8] developed a system that identifies bends from a CADmodel and uses case-based reasoning to determine the best bending sequence. Abedini et al. [9] automated the sequencing of bending operations with fuzzy set theory and later a system for sequencing punching operations with a main set partitioning (MSP) was suggested [10] where the punches are partitioned into the stations of the die with the help of priority sequences that the designer has to provide. Lin and Sheu [11] developed a method for sequencing of punches using clustering of punches to do a modified exhaustive search and then scoring of the variants. This was then used in [12] to make the complete layout planning of both punching and bending operations. According to Moghaddam et al. [13] the previous work on sequencing had not taken simultaneous operations and tolerances of specific features into account in a good enough way. So, they continued developing the system already developed in [14] that did the sequencing of both bending and punching using fuzzy set theory.

\subsection{Simulation and analysis}

In simulating and predicting formability, springback, wear, etc. many commercial software are readily availible such as AutoForm, PAM-STAMP and many other. The recent research in the area is focused on solving some specific challenges in the process. Pilthammar et al. [15] suggests a method for considering the elastic deformations of dies in order to get more accurate pressure distributions for the friction model and shape predictions i.e. draw-in and strains. This is done by first carrying out a structural FE-simulation of the tool and then transferring the deformed die shapes to the sheet metal forming simulation. The use of sub-modelling is recommended as the way to transfer the deformed shapes. To increase the accuracy of springback analysis without greatly increasing the solution time of the FE-models, Iwata and Iwata [16] proposed a method where a refined model of the drawbead and die shoulder is made separately and the results saved in a database. The data is then combined with the results of a conventional forming analysis and used to get a more accurate springback analysis.

Much research has been done within the area of optimization for the dies in SFM. Karen et al. [17] also recognizes that the deflections of the die as well as the press table 
are important to consider. To optimize the structure of the die in an efficient way they developed a new optimization algorithm where they enhanced the differential evolution method by using the best vectors as differential vectors, thus shortening the solution time. The use of load mapping from a forming simulation to get accurate forces acting on the die during the topology optimization of the tool structure was explored in [18]. A new load mapping method was proposed in order to seed up the optimization iterations. This method also considers the deflections of the die structure.

The importance to accurately model friction in SMF has been acknowledged by many. An advanced model of the friction behavior in SMF was developed by Hol et al. in [19], [20]. It accounts for the change in surface topography during the forming process and the evolution of friction from adhesion and ploughing in the boundary as well as the hydrodynamic properties of the lubricant itself. It has since been commercialized in the software TriboForm. Another frictional model implemented in Abaqus [21] takes contact pressure, slip-rate and temperature into account. The implementation also makes it possible for die designers to visualize the coefficient of friction (COF) at different stages of the forming process to make better decisions about local surface treatment of the die.

\subsection{Platform approaches}

There has been research in the past years covering platform approaches, product configurability and standardization in the context of companies with an ETO strategy. Gepp et al. [22] studied the benefits and challenges of using standardization, modularization and platform approaches in ETO companies acting in design and construction of industrial plants. The main challenges are (1) the definitions of relevant key performance indicators for tracking the success and controlling the implementation of the new system. (2) The evaluation of cost and savings from these approaches as the costs and gains usually don't occur at the same organizational locations and the savings are made over longer periods of time, and (3) the lack of methodological support for implementing these approaches.

A platform approach for companies with an ETO strategy, termed design platform (DP), was developed in [23]. It addresses many of the challenges of applying a platform approach to one-of-a-kind products by incorporating many different types of design assets. This is done in order to facilitate the standardization, modularization and platform thinking for processes, synthesis resources, product constructs, assessment resources, solutions and projects, expanding the traditional product platform from [24]. The implementation was done in connection to a PDM-system environment, linking generic product concepts and processes. Raudberget et al. extended the DP by adding support to the early stages of development [25]. By using a Set-based approach to define enhanced function-means tree, different architectural options can be created. These can then be analyzed using clustering of design structure matrices to find suitable modules that have low numbers of external functional relations. These are good candidates for flexible modules that can be changed over time with minimal impact on the rest of the system.

Johnsen et al. [26] proposes a five-step framework for improving modularity in product platforms for ETO companies to improve configurability while keeping the desired flexibility to customize. The framework uses historical data of customizations of a product as well as predictions for the future to identify potential improvement of product modularity. 


\subsection{Sensors and monitoring}

According to Kolhatkar and Pandey [1] "the sheet metal tooling industry has just entered an era of understanding and transitions into the method of bringing in intelligence in the tool". The research reviewed could be categorized into three areas. (1) Measurements of the produced parts in the form of variations in dimensions from spring back, thickness variations from material flow, unwanted wrinkling or tearing and form errors or surface defects. (2) Measurements of in order to determine tool wear, breakage or chipping. (3) Measurements of process conditions during production in the form of forces, strains, friction, temperatures, vibrations, electrical current, acoustics, etc. Some of the possibilities that increased measuring in the SMF process provide is the move from preventive maintenance to predictive maintenance and getting better knowledge and control of capability and efficiency in production.

\section{Study of industrial practice}

In this study semi-structured interviews with open ended questions were conducted at six Swedish companies (denoted C1-C6) and a total of fourteen employees included. The questions were structured to first describe the process of developing the tools, what design supports are used and motivations for them, how time and cost are distributed in the process for different tasks and then to identify challenges. At each company, the roles of tooling department manager and tool designer were included to get different internal views. The answers were audio recorded and the analysis consisted in categorizing the answers and summarizing them.

C1-C3 are tooling companies with 20-30 employees. C4 and C5 are OEMs and have internal tooling departments and manufacturing. The sites involved in this study have approximately 300 employees. C6 acts as a sub-supplier specialized in manufacturing sheet metal parts with an internal tooling and maintenance department and approximately 200 employees. The manufacturing of the tools, it is mostly done inhouse for all the companies. The machinery used consists of machining centers, milling machines, wire EDM machines, grinding machines and hardening furnace. Most of the hardening is done externally.

\subsection{Tool development process}

There are two clear categories when it comes to the tool development process. The companies that acts as sub-suppliers (C1-C3 and C6) and those that are OEMs with an internal tooling department ( $\mathrm{C} 4$ and $\mathrm{C} 5$ ). From the interviews, the processes used by the two groups are summarized and shown in Figure 1 and 2 respectively. The main differences between the groups are in the early stages of the process. From step 5 and forward, in the figures the processes are very similar. The differences are (1) that the OEMs tooling designers are involved earlier in the product development process that those at the tooling companies, (2) that the tooling companies have to answer many requests for quotations (RfQ) and (3) that the OEMs in this study have the possibility to conduct the testing in step 12 in production presses while the sub-suppliers conduct their testing in test-presses in-house. 


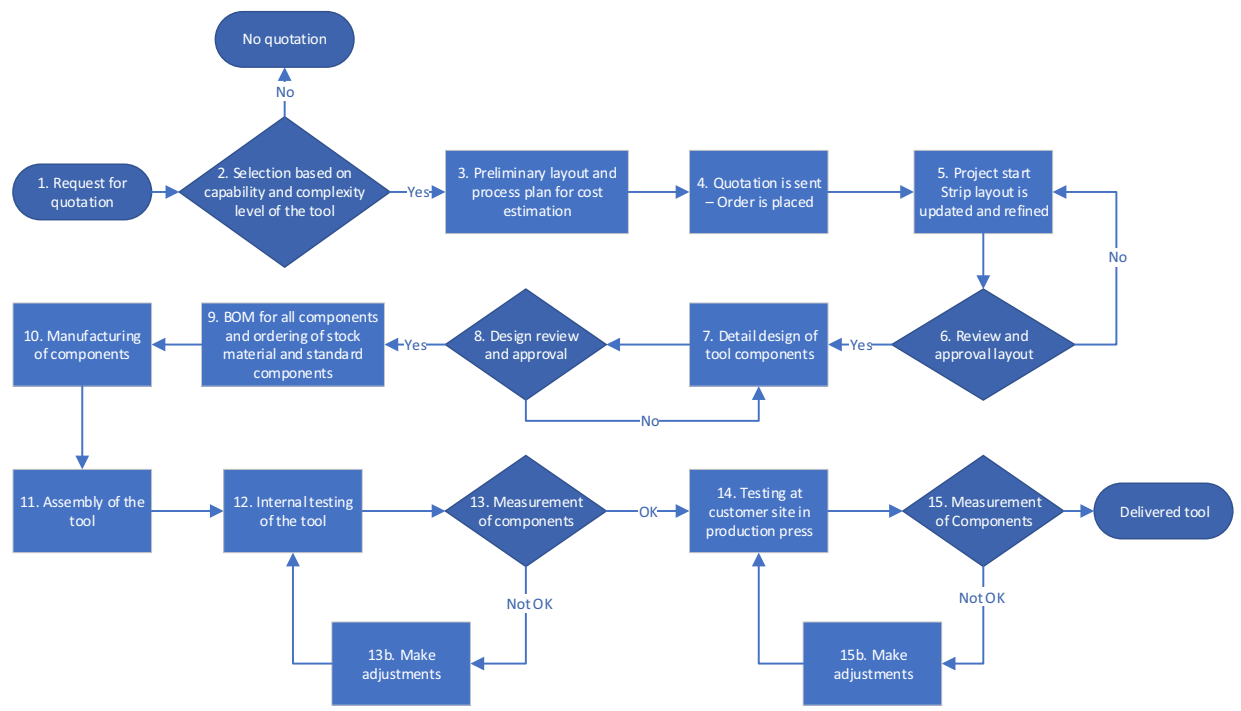

Figure 1. General development process for C1, C2, C3 and C6 (sud-suppliers).

The process in Figure 1 is representative for the sub-supplier companies but some companies differ slightly in some of the steps.

$\mathrm{C} 1$ : has a structured project management model. A project startup meeting is held in step 5 to discuss timeframe, resources and lessons learnt from similar projects with representatives from design, manufacturing and sales. The internal testing in step 12 is made in a press capable of running at production speed to evaluate the influences of that on the quality of the parts manufactured.

C6: As a manufacturing company they buy many of their tools from tooling companies. When they receive a RfQ for manufacturing of a component they, in turn, send multiple RfQs to different tooling companies. But when they develop a tool inhouse, the process in Figure 1 is representative.

For $\mathrm{C} 4$ and $\mathrm{C} 5$ and the OEM category, Figure 2 describes the general process. As they are involved earlier in the product development process, they can gain more knowledge from discussions with the product designers and creating prototypes. There are some slight differences in some steps.

C4: has multiple checkpoints in step 7 with designers and project leader.

C5: differs from the process in that the tooling department is not as involved in prototyping as no prototype tools are developed. That means they have less emphasis on step 3 .

\subsection{Time and cost distribution}

The data collected for time and cost distribution in tooling projects are presented in total for all companies as the min-max range and averages in parentheses in Table 1 and Table 2. The time spent in a typical tooling project from the point of received order or locked design is usually logged at the companies in the following categories: early cost estimation, design, manufacturing, assembly and testing. 


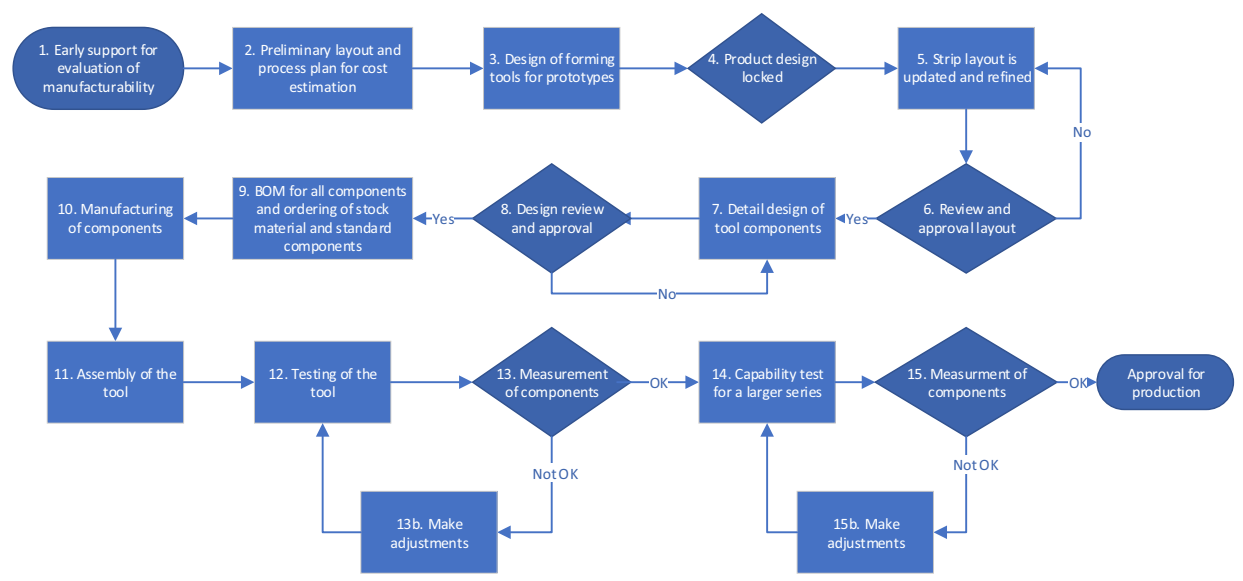

Figure 2. General development process for C4 and C5 (OEMs).

The time for making early cost estimations in step 3 for sub-suppliers and step 2 for OEMs (including estimations for lead time, scrap rate, tool size and technical solutions) is not included in the total time of a project as it is made before the project start. It ranges from $4-8 \mathrm{~h}$ depending on the complexity of the component and if similar tools have been made in the past. For $\mathrm{C} 1-\mathrm{C} 3$ the hit rate for the quotations they send out can be as low as $2-5 \%$ when they work with manufacturing companies where there is a quotation chain. When working with companies who manufacture their own products the hit rate can be $15-20 \%$. For C4 and C5 the early involvement has the effect that cost estimations must be updated following design changes of the products.

Table 1. Time distribution in \% of total time spent for categoies of activities in a stamping tool project.

\begin{tabular}{cccc}
\hline & Design & Manufacturing & Assembly/Testing \\
\hline Average & $16 \%$ & $58 \%$ & $17 \%$ \\
Min-max & $7-21 \%$ & $53-76 \%$ & $8-26 \%$ \\
\hline
\end{tabular}

Time spent on design and manufacturing depends on the size of the tool and the complexity of the sheet metal component and number of components in the tool. The number of test loops in a project can range from 1-6 and take from 2-6h each and involves multiple persons and mostly results in design changes.

The cost distribution in a tool project is broken down at the companies in the following categories: bought material (stock material and standard components), design, and manufacturing (including assembly) and is shown in Table 2.

Table 2. Cost distribution in \% of total cost for categoies in a stamping tool project.

\begin{tabular}{cccc}
\hline & Design & Material & Manufacturing \\
\hline Average & $16 \%$ & $30 \%$ & $57 \%$ \\
Min-max & $10-20 \%$ & $25-40 \%$ & $45-65 \%$ \\
\hline
\end{tabular}

\subsection{Supports used}

The design supports used by the companies can be categorized in the following categories: CAD (with unfolding functionality), Advanced unfolding, Forming simulation, Design automation, PDM, Quality Checklists, Project management. This is shown in Table 3. Only 2 of 6 companies use forming simulations on a regular basis. Two reasons for that according to the companies are (1) that it is not needed for their 
type of products or (2) that they do not have the know-how to operate the software. None of the companies use any type of design automation. The reason given for this is either that the task is done fast enough manually or that the software support restricts the way of working too much. None of the companies use any type of PDM-system for their SMF-tools. The reason for this was unanimous and was said to be that common PDM-systems are used for file permission and references and revision handling and that is not needed for them. Checklists and project management tools are sparsely, but the companies not using it, all had the ambition to start using it.

Table 3. Design supports used by the companies.

\begin{tabular}{lllllll}
\hline Support type & C1 & C2 & C3 & C4 & C5 & C6 \\
\hline CAD & Yes & Yes & Yes & Yes & Yes & Yes \\
\hline Advanced unfolding & Yes & Yes & Yes & Yes & Yes & Yes \\
\hline Forming simulation & Yes & No & No & Yes & No & No \\
\hline Design automation & No & No & No & No & No & No \\
\hline PDM & No & No & No & No & No & No \\
\hline Checklists & Yes & No & No & Yes & No & Yes \\
\hline Project management & Yes & No & No & No & No & Yes \\
\hline
\end{tabular}

\section{Analysis and identification of challenges}

From the discussions with the companies the challenges and future research suggestions described in the following sections were identified.

When analyzing the tool development process for the sub-suppliers, described earlier, one of the first steps is to answer a RfQ. To do that, a lot of the planning and major decisions for the tool design must be made at that point. This becomes a problem when the hit rates for orders are low. Then less time can be put into these tasks. The consequences are that the accuracy of the estimations decreases and that the risk of errors increases. This results in either over or under estimation of costs and in both cases the sub-supplier is affected negatively economically. It is noteworthy that few supports to automate and simulate parts of that process (as described in chapter 1) are used by the companies in this study. The systematic reuse of CAD-models, documentation and knowledge from previous projects is also low. The lack of PDM systems makes the information about previous projects only available in the minds of the designers involved. The challenge is to structure the knowledge and methods used in way so that the designers are aided by it in their work, and not restricted (as stated in chapter 2.3). Research analyzing the gap between the methods and technology that exist and what is used in practice and what is needed to bridge the gap is needed. The concepts of platforms have also been researched on a general level for ETO companies, but more research, specific to this industry is needed. For example, how supporting systems and design aids needs to be designed and structured to be adopted and to provide the right information, in the right format, at the right time to aid the designer.

The late stages in the tool development process involves testing of the tool. Drawing the right conclusions and making the correct adjustments from the test results is a critical step. $\mathrm{C} 1$ expressed that "an extra test loop can cost the entire profit margin 
of a project". The use of simulations can make this easier. Another challenge identified and expressed by the companies is that feedback from the testing should be used, not only for making changes to the current tool but also systematically formalizing and storing the knowledge to be used in coming tooling projects. Finding appropriate methods and frameworks for how to enable this would have positive impact.

The OEMs in the study expressed that there are few clear economic incentives, that they currently track, to allow them to prioritize higher quality and more reliable tools for a slightly higher initial cost. Production volumes are often increased from the initial estimations made in product development which might require higher quality material or different technical solutions in the tool. Increasing the knowledge about the SMF-process by monitoring and measuring process parameters has potential to open new opportunities in production and in tool design. Four of the companies have investigated if the use of sensors could be of interest and found that monitoring factors that are connected to accidents, e.g. press force, heat generation and power consumption in the press, have good potential to increase knowledge about the tool.

From the review of the literature of SMF simulations the research done in this area is mostly focused on getting better predictability of the final shape of the components in terms of draw-in and springback to compensate tool dies. Simulation of process robustness for SMF is a research area with a low degree of exploration. It is shown that tribological factors are important for proper predictions [19], [20]. However, C6 expressed that variations in thickness of the coil feeding into the press can be larger that the tolerances for the manufactured component and is not considered with current methods. Models considering these variations could be used to, more accurately, predict the quality of the components.

\section{Conclusions}

In this study the development processes of sheet metal stamping tools for six companies were mapped, time and cost distribution, and supports used were investigated. From the results and analysis three main challenge areas were identified. To some degree these fit within the category of knowledge capturing and reuse. This points in the direction of efforts needed in supporting the formalization and reuse of information and knowledge from previous tool projects and production, especially during the critical steps of tool process planning and creating the tool layout. There have been attempts to solving these challenges with different approaches. However, finding a good framework that ties the different aspects together in practice still requires research. Future work will investigate, in further detail, what types and forms of knowledge are reused today in the process and what knowledge is needed and useful for completing different tasks. Also, evaluating the applications of frameworks for knowledge reuse proposed in literature to this industry's challenges.

\section{References}

[1] V. Krishnan, S. D. Eppinger, and D. E. Whitney, A model-based framework to overlap product development activities, Management science, vol. 43, 1997, pp. 437-451.

[2] L. T. M. Blessing and A. Chakrabarti, DRM: A design reseach methodology. Springer, Berlin, 2009.

[3] I. Suchy, Handbook of die design, vol. 2. McGraw-Hill New York, 2006.

[4] A. Kolhatkar and A. Pandey, Sheet metal tooling: Selective review of online monitoring trends, 
International Journal of Mechanical and Production Engineering Research and Development, vol. 9, 2019, pp. 183-198.

[5] S. Kumar and R. Singh, An automated design system for progressive die, Expert Systems with Applications, vol. 38, 2011, pp. 4482-4489.

[6] M. Ghatrehnaby and B. Arezoo, A fully automated nesting and piloting system for progressive dies, Journal of Materials Processing Technology, vol. 209, 2009, pp. 525-535.

[7] M. J. Moghaddam, M. A. Farsi, and M. Anoushe, Development of a new method to automatic nesting and piloting system design for progressive die, International Journal of Advanced Manufacturing Technology, vol. 77, 2015, pp. 1557-1569.

[8] J. Y. Li, A. Y. C. Nee, and B. T. Cheok, Integrated feature-based modelling and process planning of bending operations in progressive die design, International Journal of Advanced Manufacturing Technology, vol. 20, 2002, pp. 883-895.

[9] V. Abedini, M. Shakeri, and B. Arezoo, Computer-Aided of bending progressive die design using fuzzy set theory, vol. 66 AINSC. 2010, pp. pp 657-671.

[10] M. Ghatrehnaby and B. Arezoo, Automatic strip layout design in progressive dies, Journal of Intelligent Manufacturing, vol. 23, 2012, pp. 661-677.

[11] A. C. Lin and D. K. Sheu, Computer-aided process planning of strip layout in progressive dies, Applied Mechanics and Materials, vol. 110-116, 2012, pp. 5106-5110.

[12] A. C. Lin and D. K. Sheu, Sequence planning for bending operations in progressive dies, International Journal of Production Research, vol. 50, 2012, pp. 7493-7521.

[13] M. J. Moghaddam, M. R. Soleymani, and M. A. Farsi, Sequence planning for stamping operations in progressive dies, Journal of Intelligent Manufacturing, vol. 26, 2015, pp. 347-357.

[14] J. H. Kim, C. Kim, and Y. J. Chang, Development of a process sequence determination technique by fuzzy set theory for an electric product with piercing and bending operation, International Journal of Advanced Manufacturing Technology, vol. 31, 2006, pp. 450-464.

[15] J. Pilthammar, M. Sigvant, and S. Kao-Walter, Introduction of elastic die deformations in sheet metal forming simulations, International Journal of Solids and Structures, vol. 151, 2018, pp. 7690 .

[16] T. Iwata and N. Iwata, A novel approach of springback analysis using a drawbead and a die shoulder database in sheet metal forming simulation, International Journal of Advanced Manufacturing Technology, vol. 95, 2018, pp. 3535-3547.

[17] İ. Karen, N. Kaya, and F. Öztürk, Intelligent die design optimization using enhanced differential evolution and response surface methodology, Journal of Intelligent Manufacturing, vol. 26, 2015, pp. $1027-1038$.

[18] H. Wang, H. Xie, Q. Liu, Y. Shen, P. Wang, and L. Zhao, Structural topology optimization of a stamping die made from high-strength steel sheet metal based on load mapping, Structural and Multidisciplinary Optimization, vol. 58, 2018, pp. 769-784.

[19] J. Hol, V. T. Meinders, M. B. De Rooij, and A. H. Van Den Boogaard, Multi-scale friction modeling for sheet metal forming: The boundary lubrication regime, Tribology International, vol. 81, 2015, pp. 112-128.

[20] J. Hol, V. T. Meinders, H. J. M. Geijselaers, and A. H. Van Den Boogaard, Multi-scale friction modeling for sheet metal forming: The mixed lubrication regime, Tribology International, vol. 85, 2015, pp. 10-25.

[21] F. Klocke, D. Trauth, A. Shirobokov, and P. Mattfeld, FE-analysis and in situ visualization of pressure-, slip-rate-, and temperature-dependent coefficients of friction for advanced sheet metal forming: development of a novel coupled user subroutine for shell and continuum discretization, International Journal of Advanced Manufacturing Technology, vol. 81, 2015, pp. 397-410.

[22] M. Gepp, M. Foehr, and J. Vollmar, Standardization, modularization and platform approaches in the engineer-to-order business-Review and outlook, 10th Annual International Systems Conference, SysCon 2016 - Proceedings, 2016, pp. 1-6.

[23] S. André, F. Elgh, J. Johansson, and R. Stolt, The design platform-a coherent platform description of heterogeneous design assets for suppliers of highly customised systems, Journal of Engineering Design, vol. 28, 2017, pp. 599-626.

[24] D. Robertson and K. Ulrich, Planning for Product Platforms, Sloan management review, vol. 39, 1998, pp. 19-31.

[25] D. S. Raudberget et al., Supporting design platforms by identifying flexible modules, in Proceedings of the International Conference on Engineering Design, ICED, vol. 3, 2017, pp. 191200.

[26] S. H. Markworth Johnsen, K. Kristjansdottir, and L. Hvam, Improving product configurability in ETO companies, Proceedings of the International Conference on Engineering Design, ICED, vol. 3, 2017, pp. 221-230. 